\title{
Abordagem Fisioterapêutica em um Indivíduo com Dermatopolimiosite e Artrite Reumatoide: Relato de Caso
}

\author{
Willian Guerra de Lima1, Bruna Knob², Fernanda Lorenzet Bresolin ${ }^{3}$ \\ Letícia Aparecida Bolzan", Dáfne dos Santos Ribeiro ${ }^{5}$, Caroline Zanin ${ }^{6}$ \\ Matheus Santos Gomes Jorge ${ }^{7}$, Lia Mara Wibelinger ${ }^{8}$
}

\section{RESUMO}

Introdução: A dermatopolimiosite e a artrite reumatoide são doenças reumáticas que podem cursar em concomitância, causando dor e impacto no equilíbrio postural e na qualidade de vida dos indivíduos acometidos. Objetivo: O objetivo do presente estudo é verificar os efeitos da fisioterapia em um indivíduo com dermatopolimiosite e artrite reumatoide. Materiais e métodos: Trata-se de um estudo de caso com uma mulher de 49 anos, com diagnóstico de dermatopolimiosite há 25 anos e de artrite reumatoide há 23 anos. A intervenção fisioterapêutica totalizou 30 sessões: cinesioterapia (19 sessões) e hidrocinesioterapia (11 sessões). Foram realizados exercícios de alongamento muscular, de fortalecimento muscular, de terapia manual, de propriocepção, de equilíbrio postural e de marcha. Resultados: Houve diminuição da dor em três pontos (escala visual analógica), aumento do equilíbrio postural em cinco pontos (escala de equilíbrio de Berg) e melhora da qualidade de vida nos aspectos capacidade funcional, limitação por aspectos emocionais, aspectos sociais, dor, estado geral de saúde e vitalidade (questionário de qualidade de vida SF-36). Conclusão: O plano fisioterapêutico proposto foi benéfico na dor, no equilíbrio postural e na qualidade de vida em um indivíduo com dermatopolimiosite e artrite reumatoide.

Palavras-chave: Dermatomiosite. Polimiosite. Artrite reumatoide. Fisioterapia. Hidroterapia.

\section{PHYSIOTHERAPEUTIC APPROACH IN AN INDIVIDUAL WITH DERMATOPOLYMYOSITIS} AND RHEUMATOID ARTHRITIS: CASE REPORT

\section{ABSTRACT}

Introduction: Dermatopolymyositis and rheumatoid arthritis are rheumatic diseases that can occur in concomitance, cause pain and impact on postural balance and quality of life of affected individuals. Aim: The objective of the present study was to verify the effects of physiotherapy in an individual with dermatopolymyositis and rheumatoid arthritis. Materials and Methods: It is a case study, woman, 49 years old, with diagnosis of dermatopolymyositis at 25 years and of rheumatoid arthritis at 23 years. Physiotherapy intervention consisted of 30 sessions of kinesiotherapy (19 sessions) and hydrokinesiotherapy (11 sessions). Were performed exercises of Muscle stretching, muscle strengthening, manual therapy, proprioception, postural balance and gait. Results: There was a decrease in pain in three points (visual analogue scale), increase of the postural balance in five points (Berg balance scale) and improvement of the quality of life in aspects functional capacity, limitation by emotional aspects, social aspects, pain, general health and vitality (questionnaire quality of life SF-36). Conclusion: The proposed physiotherapeutic plan was beneficial in pain, postural balance and quality of life in an individual with dermatopolymyositis and rheumatoid arthritis. Keywords: Dermatomyositis. Polymyositis. Rheumatoid arthritis. Physical Therapy specialty. Hydrotherapy.

\footnotetext{
Graduando do curso de Fisioterapia, Faculdade de Educação Física e Fisioterapia, Universidade de Passo Fundo, bolsista Pibic/CNPq, Passo Fundo (RS), Brasil. willianguerralima@hotmail.com

2 Graduanda do curso de Fisioterapia, Faculdade de Educação Física e Fisioterapia, Universidade de Passo Fundo, Passo Fundo (RS), Brasil. brunaknob33@gmail.com

${ }^{3}$ Graduanda do curso de Fisioterapia, Faculdade de Educação Física e Fisioterapia, Universidade de Passo Fundo, Passo Fundo (RS), Brasil. fernanda-bresolin@outlook.com

${ }^{4}$ Graduanda do curso de Fisioterapia, Faculdade de Educação Física e Fisioterapia, Universidade de Passo Fundo, Passo Fundo (RS), Brasil. leticiabolzan57@gmail.com Graduanda do curso de Fisioterapia, Faculdade de Educação Física e Fisioterapia, Universidade de Passo Fundo, Passo Fundo (RS), Brasil. daafne.ribeiro@gmail.com ${ }^{6}$ Graduanda do curso de Fisioterapia, Faculdade de Educação Física e Fisioterapia, Universidade de Passo Fundo, Passo Fundo (RS), Brasil. carolczanin@gmail.com Fisioterapeuta e pós-graduando do curso de Especialização em Fisioterapia Traumato-ortopédica, Faculdade de Educação Física e Fisioterapia, Universidade de Passo Fundo, Passo Fundo (RS), Brasil.mathjorge5@gmail.com

${ }^{8}$ Doutora em Gerontologia Biomédica pela Pontifícia Universidade Católica, Porto Alegre (RS), Brasil. Docente do curso de Fisioterapia e do Programa de Pós-Graduação em Envelhecimento Humano, Faculdade de Educação Física e Fisioterapia, Universidade de Passo Fundo, Passo Fundo (RS), Brasil. liafisio@yahoo.com.br
} 


\section{INTRODUÇÃO}

A dermatopolimiosite (DPM) é uma doença reumática idiopática, de caráter crônico inflamatório, autoimune e não supurativo. Suas principais características são as alterações musculoesqueléticas, tais como a diminuição da força muscular, da resistência, da capacidade aeróbica, o aumento da fadiga, mialgias e outras manifestações sistêmicas (cardíaca, pulmonar, vascular e gastrointestinal). A inflamação gerará disfunção na força muscular e importantes alterações metabólicas, como a diminuição da capacidade de oxidação, resultando em um déficit funcional (WIBELINGER, 2014; BERTOLUCCl et al., 2014; ALEMO MUNTERS et al., 2013a).

A artrite reumatoide (AR), por sua vez, é uma doença crônica, inflamatória e multissistêmica, também de ordem reumática. Caracteriza-se pelo envolvimento articular, especialmente das pequenas articulações, como as das mãos e pés. Seu quadro clínico é acompanhado de sinovite inflamatória, edema e erosões articulares e periarticulares. A rigidez matinal e a dor são os sintomas mais comumente encontrados entre seus acometidos, podendo manifestar-se de forma simétrica e gerar graves comorbidades (WIBELINGER, 2014; OTTAWA, 2015; PARK; CHANG, 2016).

O exercício físico nas doenças reumáticas tem sido visto como importante no desenvolvimento da terapia, por produzir efeitos benéficos no desempenho muscular, na capacidade aeróbica e na saúde de forma geral de indivíduos com DPM e AR (GUALANO et al., 2011; ALEMO MUNTERS et al., 2014; OTTAWA, 2015). Na DPM, a fisioterapia é efetiva tanto na recuperação de danos quanto na prevenção do surgimento das contraturas e alterações posturais. De forma semelhante, na AR a fisioterapia desempenha importante função em todos os estágios da patologia, desde a prevenção de deformidades até a amenização dos prejuízos causados (WIBELINGER, 2014).

Neste sentido, o objetivo deste estudo é verificar os efeitos da fisioterapia em um indivíduo com DPM e AR.

\section{MATERIAIS E MÉTODOS}

Este é um estudo de caso que faz parte de um projeto guarda-chuva denominado "Efeitos do tratamento fisioterapêutico em pacientes portadores de doenças reumáticas", aprovado pelo Comitê de Ética em Pesquisa em Seres Humanos da Universidade de Passo Fundo, sob protocolo no 348.381, conforme de- termina a Declaração de Helsink de 1975, revisada em 2000, e a Resolução do Conselho Nacional de Saúde do Brasil no 466/2012.

Participou do estudo um indivíduo do gênero feminino, 49 anos, solteira. Aos 24 anos apresentou sintomas de perda de força em membros superiores e inferiores. Após dois anos, recebeu o diagnóstico clínico de DPM e, posteriormente, AR. Não apresenta histórico familiar da doença reumática e possui insuficiência venosa crônica em associação às demais patologias. Faz uso de seis medicamentos contínuos e manteve a terapia medicamentosa durante o tratamento fisioterapêutico.

O indivíduo chegou ao Serviço de Fisioterapia Reumatológica da Universidade de Passo Fundo com queixas de perda de força muscular, dores do tipo "agulhadas" nos membros inferiores e na coluna vertebral, além de dificuldade em realizar Atividades de Vida Diária. O laudo fisioterapêutico constatou redução da amplitude de movimento articular e da força muscular generalizada.

O indivíduo foi avaliado, pré e pós intervenção, quanto à dor por meio da escala visual analógica, quanto ao equilíbrio por meio da escala de equilíbrio de Berg e quanto à qualidade de vida por meio da versão brasileira do questionário de Qualidade de Vida SF-36.

A escala visual analógica da dor é uma linha reta horizontal com suas extremidades numeradas de zero (nenhuma dor) a 10 (pior dor imaginável) (MARTINEZ; GRASSI; MARQUES, 2011). A escala de equilíbrio de Berg foi desenvolvida para avaliar o equilíbrio postural. Sua pontuação máxima é de 56 pontos e possui em cada item uma escala ordinal de 5 alternativas que variam de zero a 4 pontos. Quanto maior a pontuação obtida melhor é o equilíbrio do indivíduo (BERG et al., 1992). O questionário de qualidade de vida SF-36 é uma escala composta por 11 perguntas, abrangendo domínios como capacidade funcional, aspectos físicos, dor, estado geral de saúde, vitalidade, aspectos sociais, aspectos emocionais e saúde mental. O escore final varia de zero (pior estado geral de saúde) a cem (melhor estado geral de saúde) (BURILLE et al., 2012).

Mediante estes achados foram traçados objetivos, tais como o alívio da dor, a manutenção ou aumento da amplitude de movimento articular, o aumento do trofismo muscular e a melhora da capacidade cardiopulmonar do indivíduo. Para isso, os exercícios consistiram em alongamento ativo assistido do tronco, dos membros superiores e dos inferiores, fortalecimento muscular, exercícios respiratórios e mobilizações articulares. 
O tempo de intervenção foi de 30 sessões durante 18 semanas, com 19 sessões de cinesioterapia e 11 de hidroterapia.

Em solo foram realizados alongamentos ativos assistidos de membros superiores e inferiores, coluna cervical, torácica e lombar; exercícios ativos de amplitude de movimento dos membros inferiores; terapia manual (massoterapia nos pés, pompagem miofascial e liberação diafragmática); mobilização articular de tornozelos e punhos, tração de punho; técnicas de Facilitação Neuromuscular Proprioceptiva nas diagonais de flexão-abdução-rotação externa; exercícios respiratórios; exercícios proprioceptivos e de equilíbrio; treino de marcha cruzada, com obstáculos e transferência de peso.

Em meio aquático, foram realizados fortalecimento dos músculos extensores, flexores, abdutores e adutores do quadril, flexores e extensores do joelho, abdutores, flexores e extensores do ombro, flexores e extensores do cotovelo, flexores e extensores de punho e dedos, e flexores, extensores e rotadores de tronco.

Para isso foram utilizados os seguintes materiais: combinação de exercícios isotônicos e isométricos, bolas suíças, faixas elásticas, bastões, fortalecedores e exercitadores de dedos, dispositivos para treino de equilíbrio, caneleiras e halteres.

\section{RESULTADOS}

A Tabela 1 apresenta os resultados referentes à intensidade da dor do indivíduo estudado nas fases pré e pós intervenção fisioterapêutica.

Tabela 1 - Dor pré e pós intervenção fisioterapêutica

\begin{tabular}{lcc}
\cline { 2 - 3 } & Pré intervenção & Pós intervenção \\
\hline Dor & 7 pontos & 4 pontos \\
\hline
\end{tabular}

Houve diminuição da intensidade da dor em três pontos de acordo com a escala visual analógica.

A Tabela 2 apresenta os resultados referentes ao equilíbrio postural do indivíduo estudado nas fases pré e pós intervenção fisioterapêutica.

Tabela 2 - Equilíbrio postural pré e pós intervenção fisioterapêutica

\begin{tabular}{lcc} 
& Pré intervenção & Pós intervenção \\
\hline Pontuação & & \\
da escala de & 42 pontos & 47 pontos \\
equilíbrio de Berg & & \\
\hline
\end{tabular}

Observou-se aumento do equilíbrio postural muscular em cinco pontos de acordo com a escala de equilíbrio de Berg.
A Tabela 3 apresenta os resultados referentes à qualidade de vida do indivíduo estudado nas fases pré e pós intervenção fisioterapêutica.

Tabela 3 - Qualidade de vida pré e pós intervenção fisioterapêutica

\begin{tabular}{lcc}
\hline Domínios & $\begin{array}{c}\text { Pré } \\
\text { intervenção }\end{array}$ & $\begin{array}{c}\text { Pós } \\
\text { intervenção }\end{array}$ \\
\hline Capacidade funcional & 35 & 70 \\
Limitação por aspectos físicos & 50 & 25 \\
Dor & 10 & 20 \\
Estado geral de saúde & 20 & 30 \\
Vitalidade & 50 & 55 \\
Aspectos sociais & 50 & 62,5 \\
Limitação por aspectos emocionais & 66,66 & 100 \\
Saúde mental & 60 & 52 \\
\hline
\end{tabular}

Em linhas gerais, houve melhora em seis dos oito domínios avaliados que constituem a qualidade de vida, sendo eles a capacidade funcional, a limitação por aspectos emocionais, os aspectos sociais, a dor, o estado geral de saúde e a vitalidade, respectivamente.

\section{DISCUSSÃO}

Indivíduos com desordens reumáticas possuem uma reabilitação que consiste em um processo diário, com envolvimento de várias etapas e de forma multidisciplinar. Sua participação em programas de exercícios regulares efetua-se com segurança, com amplas possibilidades práticas, as quais requerem considerações específicas acerca das características de cada processo patológico e do indivíduo isoladamente (WIBELINGER, 2014).

No tratamento da DPM, obtiveram-se efeitos benéficos a partir da realização de terapia com o envolvimento de reforço muscular, capacidade aeróbica e na saúde geral. Dentre os resultados obtidos, também estão a melhora da força muscular, capacidade aeróbica (pela ativação de fatores gênicos, os quais interferem na função mitocondrial e na morfologia vascular) e a redução de genes ligados à resposta inflamatória, reduzindo a atividade patológica (ALEMO MUNTERS et al., 2014), o que vai ao encontro do presente estudo, que evidenciou melhora da qualidade de vida do indivíduo, uma vez que esta variável está relacionada a diversos aspectos físicos e psicológicos do ser humano.

Além disso, o exercício contribui de forma sistêmica, mostrando-se fundamental para o ganho de desempenho muscular e saúde como um todo. Os indivíduos que realizaram essa prática mantiveram seu ganho muscular por um ano, evidenciando-se a importância da inclusão de exercícios físicos de resis- 
tência na manutenção da saúde de forma sistêmica em portadores de DPM. Ainda, tal intervenção física facilita a introdução de um estilo de vida fisicamente ativo (ALEMO MUNTERS et al., 2014). Desta forma, o tratamento fisioterapêutico parece produzir efeitos positivos a longo prazo. O tempo de intervenção do estudo foi de 30 sessões ao longo de 18 semanas e demonstrou melhoras nas variáveis dor, equilíbrio postural e qualidade de vida de um indivíduo com DPM em associação à AR.

Um programa de exercícios desenvolveu-se em torno de 12 semanas com 2 intervenções semanais, por meio da prática de exercícios com baixa resistência, semelhantes aos do presente estudo, e restrição parcial do fluxo sanguíneo. A partir dos resultados de tal terapia, observou-se a segurança e a efetividade de sua prática, assim como do aumento da força e massa muscular e da qualidade de vida relatada. Além disso, a restrição do fluxo sanguíneo obteve efeitos ainda mais significantes nos membros inferiores, relativos ao aumento da funcionalidade e força muscular. Consequentemente houve redução nas limitações em atividades diárias, ambas comumente afetada nas extremidades proximais e distais de tais indivíduos (MATTAR et al., 2014).

Os exercícios físicos produzem efeitos significantes na redução da atividade da doença mediante seu efeito anti-inflamatório. Aqueles específicos para resistência apresentam maior eficácia em comparação a outros tipos de atividades físicas para alcançar tais efeitos. Para isso, promovem melhora no $\mathrm{VO}_{2}$ máximo por ativação do nervo vago, causando uma redução da atividade da doença e um consequente aumento da força muscular (ALEMO MUNTERS et al., 2013b).

Indivíduos com DPM apresentam menores capacidades na realização de exercícios aeróbicos. Por outro lado, os que realizaram uma intervenção baseada no aumento da resistência por meio de bicicleta e subir escadas por 12 semanas, aumentaram sua capacidade aeróbica e diminuíram níveis de fadiga e produtos metabólicos (ALEMO MUNTERS et al., 2013b). Aliás, a realização de exercícios supervisionados reduz a atividade patológica de forma mais significativa, suprimindo fatores inflamatórios no sistema musculoesquelético (ALEMO MUNTERS et al., 2014), evidenciando sua aplicabilidade terapêutica.

A atividade física demonstra efeitos positivos sobre a força muscular, função, capacidade aeróbica, humor e incapacidade, beneficiando, como consequência, a qualidade de vida de portadores de AR, em concordância com os resultados obtidos neste estudo. Dentre as intervenções está a terapia de exercícios, modalidades eletrofísico, órteses e dispositivos de assistência e intervenções de autogestão (incluindo educação), proporcionando alívio sintomático sem alterar o curso da progressão da doença, melhorando a qualidade de vida (OTTAWA, 2015; IVERSEN; BRAWERMAN; IVERSEN, 2012). Isso vem ao encontro dos resultados obtidos neste estudo, visto que o indivíduo apresentava AR, além da DM.

Eletrotermoterapias, como a crioterapia e a eletroterapia, são fundamentais na AR. A crioterapia é mais indicada em períodos não agudos e com ausência do Fenômeno de Raynaud; já a eletroterapia apresenta excelente desempenho na redução do quadro álgico, redução do edema e inflamação, possibilitando futura prática de exercícios e ganho funcional (WIBELINGER, 2014). Tais terapêuticas, no entanto, não apresentaram relevância na escolha do tratamento a ponto de serem incluídas nele.

Em uma intervenção desenvolveu-se um plano de tratamento que consiste em sessões de diferentes meios terapêuticos (eletroacupuntura, massagem, aplicação de lodo e uso do calor). Sua realização efetuou-se durante 3 semanas, produzindo diminuição da dor nos resultados da Escala Visual Analógica (EVA) em aspectos psicológicos e uma melhora na qualidade de vida e capacidade física geral de indivíduos com $A R$ (SHETTY; MOOVENTHAN, ANAGHA, 2015), em consonância com os resultados das diatermias na hidrocinesioterapia.

Outro estudo comparou a aplicação na crioterapia em todo o corpo e a fisioterapia por intermédio de terapia magnética, eletroterapia e ultrassom, com ambos os tratamentos da AR aliados à exercícios físicos com duração de duas semanas. Nos resultados observou-se redução da dor de acordo com a EVA em ambos os tratamentos, com maior destaque para a melhora da saúde geral a partir da segunda terapia (GIZIŃSKA et al., 2015), evidenciando o potencial da cinesioterapia em combinação à eletrotermoterapias na AR.

\section{CONCLUSÃO}

A escolha do método terapêutico para a intervenção em patologias reumáticas requer o entendimento de um conjunto de fatores. Em casos em que há comorbidades, a escolha do tratamento requer uma consideração extra acerca de todos os fatores envolvidos em ambos os processos patológicos. No caso apresentado, o tratamento da DPM e da AR por intermédio de alongamento, fortalecimento muscular, hidroterapia, aplicação de técnicas massoterapêuticas e 
exercício aeróbico, promoveu ganho nos domínios de qualidade de vida e equilíbrio além de reduzir a dor. Dessa forma, a intervenção mostrou-se eficaz e de boa aplicabilidade no tratamento da AR em associação à DPM.

\section{REFERÊNCIAS}

ALEMO MUNTERS, L. et al. New insights into the benefits of exercise for muscle health in patients with idiopathic inflammatory myositis. Current Rheumatology Reports, v. 16, n. 7, p. 429, 2014.

. Improved exercise performance and increased aerobic capacity after endurance training of patients with stable polymyositis and dermatomyositis. Arthritis Research \& Therapy, v. 15, n. 4, p. 83, 2013 a.

. Improvement in health and possible reduction in disease activity using endurance exercise in patients with established polymyositis and dermatomyositis: a multicenter randomized controlled trial with a 1-year open extension followup. Arthritis Care \& Research, v. 65, n. 12, p. 1.959-1.968, 2013b.

BERG, K. et al. Clinical and laboratory measures of postural balance in an elderly population. Archives of Physical Medicine and Rehabilitation, v. 73, n.11, p. 1.073-1.080, 1992.

BERTOLUCCI, F. et al. Abnormal lactate levels in patients with polymyositis and dermatomyositis: the benefits of a specific rehabilitative program. European Journal of Physical and Rehabilitation Medicine, v. 50, n. 2, p. 161-169, 2014.

BURILLE, A. et al. Qualidade de vida de portadores de espondilite anquilosante submetidos a um programa de hidrocinesioterapia. Lecturas Educación Física y Deportes, v. 17, n. 169. p. 1, 2012.

GIZINSSKA, M. et al. Effects of Whole-Body Cryotherapy in Comparison with Other Physical Modalities Used with Kinesitherapy in Rheumatoid Arthritis. BioMed. Research International, v. 2.015, n. 2.015, p. 1, 2015.

GUALANO, B. et al. Therapeutic effects of exercise training in patients with pediatric rheumatic diseases. Revista Brasileira de Reumatologia, v. 51, n. 5, p. 490-496, 2011.

IVERSEN, M. D.; BRAWERMAN, M.; IVERSEN, C. N. Recommendations and the state of the evidence for physical activity interventions for adults with rheumatoid arthritis: 2007 to present. International Journal of Clinical Rheumatology, v. 7, n. 5, p. 489-503, 2012.

MARTINEZ, J. E.; GRASSI, D. C. Y.; MARQUES, L. G. Análise da aplicabilidade de três instrumentos de avaliação de dor em distintas unidades de atendimento: ambulatório, enfermaria e urgência. Revista Brasileira de Reumatologia, v. 51, n. 1, p. 304-308, 2011.

MATTAR, M. A. et al. Safety and possible effects of low-intensity resistance training associated with partial blood flow restriction in polymyositis and dermatomyositis. Arthritis Research \& Therapy, v. 16, n. 5, p. 473, 2014.
OTTAWA (Canadá). Canadian Agency for Drugs and Technologies in Health (CADTH). Golimumab (Simponi) IV. In: Combination with Methotrexate (MTX) for the Treatment of Adult Patients with Moderately to Severely Active Rheumatoid Arthritis. Ottawa, 2015.

PARK, Y.; CHANG, M. Effects of rehabilitation for pain relief in patients with rheumatoid arthritis: a systematic review. Journal of Physical Therapy Science, v. 28, n. 1, p. 304-308, 2016.

SHETTY, G. B.; MOOVENTHAN, A.; ANAGHA, N. Effect of electro-acupuncture, massage, mud, and sauna therapies in patient with rheumatoid arthritis. Journal of Ayurveda and Integrative Medicine, v. 6, n. 4, p. 295-299, 2015.

WIBELINGER, L. M. Fisioterapia em reumatologia. Rio de Janeiro: Revinter, 2014. 